\title{
Recent advancements in the "water-window" microscopy with laser-plasma SXR source based on a double stream gas-puff target
}

\author{
P.W. WACHULAK* \\ Institute of Optoelectronics, Military University of Technology, ul. gen. S. Kaliskiego 2, \\ 00-908 Warsaw, Poland
}

An overview of our recent developments, regarding "water-window" soft X-ray (SXR) microscopy based on a laser-plasma double stream gas puff target sources is presented. The work, presented herein, describes two approaches to SXR microscopy. The first one is a low spatial resolution, achromatic SXR microscopy, employing Wolter type-I objective. The second one is a nanometer spatial resolution SXR microscopy, with the use of a Fresnel zone plate objective, for imaging various objects with quasimonochromatic light, emitted from a double stream gas puff target based short wavelength source. The developments regarding both systems are presented, as well as the possible applications, for which the SXR microscope was already employed. Such compact, table-top size, laboratory type microscopy setups may be employed in the near future for complementary-like studies to other, often used, microscopy techniques.

Keywords: soft X-rays, "water window”, microscopy, Wolter optics, Fresnel zone plat-e, gas puff target, laser-produced plasma.

\section{Introduction}

All the advancements in various branches of science would not be certainly possible without the discovery and developments of the visible light microscopy. Since its discovery, it benefited biology, medicine, material science, physics, etc. allowing for major contributions in almost all physical sciences. Work done by Hans and Zacharias Janssens, Robert Hooke, Anton van Leeuwenhoek, and later by Ernst Abbe, among others, allowed finally to construct diffraction limited visible light optical systems, capable of resolving features of the order of few hundreds of nanometers. However, as the wavelength of visible radiation is between $400-700 \mathrm{~nm}$ it is a major obstacle to reach much higher spatial resolutions. One of the simplest and most direct ways to improve spatial resolution in photon-based imaging systems is decreasing the illumination wavelength, thus the widespread interest can be observed in microscopy at extreme ultraviolet (EUV) and even shorter - soft X-ray (SXR) wavelengths. Photon-based (bosonic-type) imaging at short wavelength vs. electron, or recently neutron, imaging has additional advantages due to different interaction of photons with matter. Atomic resonance frequencies, leading to very high absorption coefficients at the EUV and SXR wavelengths, provide enhanced optical contrast.

\footnotetext{
*e-mail: wachulak@gmail.com
}

Among various different wavelengths from the EUV and SXR spectral regions particularly suitable range of wavelengths for biological imaging is so called "water-window" spectral range, between 2.3 and $4.4 \mathrm{~nm}$ [1]. High contrast in this spectral range is obtained due to a difference in absorption of different constituents of biological specimen. While water, present in the sample, has relatively small absorption coefficient in this spectral range, carbon, due to much higher absorption, gives a very good contrast in the image. Thus, this spectral range is perfectly suitable for imaging of biological specimen.

In this mini-review I present our recent developments which combine the advantages of employing compact, laboratory type laser-plasma short wavelength source with the "water-window" spectral range. Such source is based on a double stream gas puff target and emits incoherent radiation. This unique combination is suitable for biological imaging, and allows developing small size microscopy setups which might be used in various fields of science and technology. I will present both, achromatic SXR microscopy system with a sub-micron spatial resolution and, as a natural extension of such system, a nanometer spatial resolution microscope. The first one employs a Wolter type-I reflective optic and a broad band "water-window" source. This system allows capturing magnified images of the objects with $\sim 1 \mu \mathrm{m}$ spatial resolution and exposure time as low as 5 seconds. It is 
benefiting from the chromatic aberration free optics, which allows changing immediately the illumination spectral region. The second system, based on a Fresnel zone plate objective, has $\sim 60 \mathrm{~nm}$ spatial resolution and operates using a quasimonochromatic emission from nitrogen based SXR source. Both systems are very compact, allowing for an easy user-friendly operation and a possible future commercialization. A detailed characterization and optimization of both microscopy setups, as well as some examples of possible applications, will be presented and discussed.

\section{Previous work}

The idea behind employing short wavelength sources for microscopy reaches back at least to XIX century, when Ernst Abbe developed his spatial resolution formula for optical systems. It states that the spatial resolution of the diffraction limited optical system is directly proportional to the illumination wavelength. It has been a long time, however, before first X-ray tubes combined with reflective optics, such as curved mirrors for example, have been used to produce images of objects, although, with inferior resolution to visible light microscopes. The development of laser plasma sources paved the way for the first demonstrations of photon-based imaging with superior resolution to the visible light diffraction limit. Since then, much work has been done in the field of high resolution imaging with both large scale facilities and compact "water-window" sources. A few examples of such work are mentioned in the following section.

Utilizing coherent illumination a $700 \mathrm{~nm}$ half-pitch resolution images with EUV recombination laser at $\lambda=18.2 \mathrm{~nm}$ has been reported in the early imaging work [2]. Better spatial resolution equal to $75 \mathrm{~nm}$ was reported utilizing a SXR laser at $\lambda=4.48 \mathrm{~nm}$, pumped by a large fusion-class NOVA laser [3]. Recently, different approaches have emerged due to the development of smaller-scale short wavelength sources such as high order harmonic generation (HHG) [4], EUV lasers [5] and incoherent laser-plasma sources [6] that have been successfully used for sub-micrometer resolution imaging. Using radiation from a table-top capillary discharge EUV laser images were obtained with a spatial resolution of 120-150 nm [7], later improved to $~ 50 \mathrm{~nm}[8,9]$. A $\lambda=13.2 \mathrm{~nm}$ wavelength radiation from Ni-like cadmium EUV laser allowed for a sub-38 $\mathrm{nm}$ resolution nano-imaging [10]. A quasi-monochromatic emission from a laser-plasma EUV source based on a gas puff target [11] allowed for $50 \mathrm{~nm}$ spatial resolution imaging $[12,13]$ in a very compact system. A quasi-monochromatic emission from an incoherent SXR source, at much shorter wavelength, $\lambda=2.88 \mathrm{~nm}$, in the "water-window" spectral range, allowed to demonstrate SXR microscopy with a sub-50 nm spatial resolution [14]. Ethanol droplet based SXR source at $\lambda=3.37 \mathrm{~nm}$, combined with a zone plate objective, allowed to capture images with spatial resolution of $60 \mathrm{~nm}$ [15], later improved to $\sim 50 \mathrm{~nm}$ [16]. Similar source was recently used to perform SXR microscopy with a $40 \mathrm{~nm}$ spatial resolution at up to $1 \mathrm{kHz}$ source repetition rate [17]. Liquid nitrogen based SXR source at $\lambda=2.48 \mathrm{~nm}$ was used to demonstrate a compact full-field soft X-ray transmission microscopy with a sub$-60 \mathrm{~nm}$ resolution, operating at $100 \mathrm{~Hz}$ repetition rate, with exposure times of less than 5 min using both dry and wet samples [18]. The same source was also used to perform SXR imaging with $\sim 40 \mathrm{~nm}$ spatial resolution and one minute exposure time for imaging of high contrast diatoms [19] and cryofrozen biological specimen. For that the source repetition rate was increased up to $2 \mathrm{kHz}$ [20], however, for the source size occupying quite a large space [21] limiting its possible future commercialization. Tomographic high resolution imaging was also performed in the "water-window" spectral range with a diatom, acquiring a tilt series of 53 images covering $180^{\circ}$ with half-period spatial resolution of the tomogram approaching $140 \mathrm{~nm}$ [22]. Using a Schwarzschild reflective objective, with a $32 x$ magnification and $\mathrm{NA}=0.2$ images of test objects were acquired with a half-pitch spatial resolution better than $0.5 \mu \mathrm{m}$ [23].

Large synchrotron facilities were also extensively utilized for the implementation of full field microscopes with record spatial resolution of $12 \mathrm{~nm}$ [24] or $14 \mathrm{~nm}$ utilizing $\lambda=1.38 \mathrm{~nm}$ undulator radiation and third order zone plate diffraction [25], also including magnetic material imaging [26-29]. Soft X-ray microscopy has been successfully employed mainly in a transmission mode, either using diffractive optics, such as zone-plates [30-32], raster scanning of the sample by focused SXR beam [33-35] or as a contact microscopy. In the contact microscopy the sample is placed on top of a recording medium, such as a photoresist, and illuminated by the SXR beam to make a "picture" (imprint) of the specimen in the surface of the recording medium [36,37]. Synchrotron radiation at $\lambda=2.4 \mathrm{~nm}$ was used for imaging frozen-hydrated samples at atmospheric pressure, where details inside cells of algae, as small as $35 \mathrm{~nm}$, were visible [38]. Also it was used to examine rapidly frozen mouse 3T3 cells and obtained excellent cellular morphology at better than $50 \mathrm{~nm}$ lateral resolution, using transmission SXR microscope [39]. Synchrotron based microscope in the "water-window" spectral range was developed to image frozen hydrated specimens with a thickness up to $10 \mu \mathrm{m}$ at temperatures of around $100 \mathrm{~K}$ [40]. Among those impressive results it is worth to notice that they were obtained either using large scale synchrotron facilities for microscopy systems, or very complicated, difficult to maintain and expensive laboratory systems, occupying a few optical tables in size. My goal is to develop much more compact microscopes, operating in the "water-window" spectral range for variety of applications, 
Recent advancements in the "water-window" microscopy with laser-plasma SXR source based on a double stream...

which provide nanometer spatial resolution and overcome presented limitations of other, larger and more complicated systems.

\section{Description of the laser-plasma SXR source based on a double stream gas puff target for microscopy}

Thus, herein, I present an overview of work, which had employed a laser-plasma source based on a double stream gas puff target. The SXR source, used in the experiment, has been developed initially for EUV metrology applications in the frame of MEDEA+ project [41]. Later it was also modified for efficient emission of SXR radiation, including the "water window" spectral region, previously reported in Ref. 42. The scheme of the source is depicted in Fig. 1(a). For the SXR microscope either argon plasma, or nitrogen plasmas were produced by focusing pumping laser pulses, from Nd:YAG laser (Eksma), with duration of 3-4 ns and energy of $0.74 \mathrm{~J}$ by a lens onto a gas puff target. Such laser-produced plasma radiates in a very broad range of wavelengths, including the SXR region. By using additional spectral filtering it is possible to tailor the spectral emission of the source according to particular needs. The source can operate up to a $10 \mathrm{~Hz}$ repetition rate. This source has a major advantage over other compact sources that it is a debris-free source and has a possibility to change the gases, thus allowing changing both the peak emission wavelength and the bandwidth of the emission.

The laser plasma source was optimized for efficient generation of SXR radiation from argon and nitrogen plasmas. Argon plasma emits a broad band radiation from the SXR region [43], while nitrogen plasma produces spectrally much

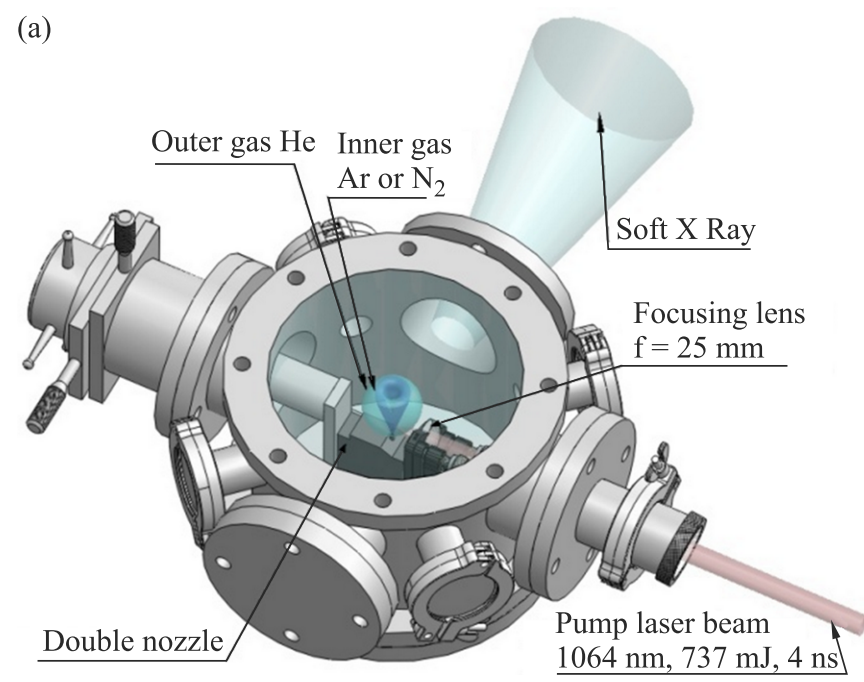

narrower, quasi monochromatic radiation, required for highly dispersive diffractive optics [44]. The targets are formed by two circularly symmetric nozzles. Such nozzles are depicted in Fig. 1(b). The inner nozzle, $0.4 \mathrm{~mm}$ in diameter, injects a small amount of working gas (argon or nitrogen) into the vacuum. The outer nozzle, ring-shaped, $0.7-1.5 \mathrm{~mm}$ in diameter, injects a low- $Z$ gas (helium). This is required to narrow down the flow of the working gas, reducing its density gradient along the nozzle axis. The nozzle axis was positioned almost concentrically with the laser focal point, displaced by a few hundreds of microns, depending on the working gas used. The displacement was in the direction opposite to the condenser optic, to reduce the re-absorption of SXR radiation in a neutral gas from the target. The distance from the focal point to the nozzle plane was $1.5 \mathrm{~mm}$ to avoid nozzle damage by repeatable pulsed plasma formation. The details about optimization were similar to one described previously in Ref. 42. One of the most important parameter to optimize, besides the gas pressures, is proper timing of the nozzles in respect to the synchronization pulse from the laser power supply. Depending on the gas used as a target the timing slightly change, which was reported previously in Refs. 43,44.

Visible light image of the plasma, created by interaction of the gas puff target with focused laser beam, is shown in Fig. 1(c). To assess the SXR spectrum of radiation emitted from that plasma a transmission grating spectrometer was used. It was equipped with 5000 -lines $/ \mathrm{mm}$ free-standing gold grating, $\sim 30 \mu \mathrm{m}$ entrance slit and a charge coupled device (CCD) camera (i-Kon M, Andor). Figure 2(a) shows a typical spectrum from an argon/helium gas puff target source. The figure shows both spectra: direct and filtered by a $200 \mathrm{~nm}$ thick titanium (Ti) filter. Thicker filter might be (b)

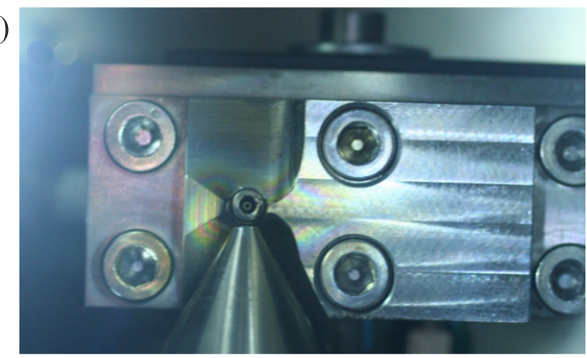

(c)

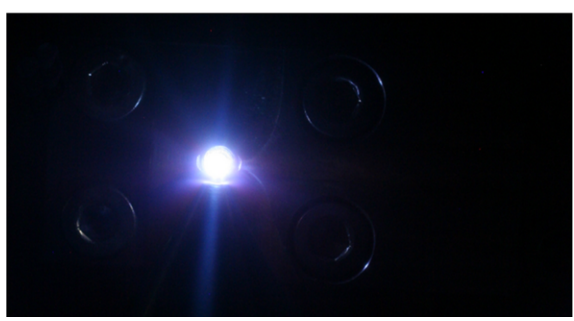

Fig. 1. Scheme of a double stream gas puff target laser plasma source (a). Photograph of the electromagnetic valve and the concentric nozzles (b) and visible light photograph of the plasma (c). 
used to narrow spectral emission from Ar plasma, however, it will limit the number of available photons for subsequent imaging and will affect the quality of the SXR image. The figure depicts Ar emission spectrum in the wavelength range from 1 to $11 \mathrm{~nm}$, with the dominant groups of argon spectral lines, such as $\mathrm{Ar}^{8+}: 2 \mathrm{~s}^{2} 2 \mathrm{p}^{6}-2 \mathrm{~s}^{2} 2 \mathrm{p}^{5} 4 \mathrm{~s}$, wavelengths between $\lambda=36.78 \AA$ and $\lambda=36.96 \AA, \mathrm{Ar}^{8+}: 2 \mathrm{~s}^{2} 2 \mathrm{p}^{6}-2 \mathrm{~s}^{2} 2 \mathrm{p}^{5} 3 \mathrm{~d}(\lambda=$ 41.48-42.56 $\AA), \mathrm{Ar}^{8+}: 2 \mathrm{~s}^{2} 2 \mathrm{p}^{6}-2 \mathrm{~s}^{2} 2 \mathrm{p}^{5} 3 \mathrm{~s}(\lambda=48.73-49.18 \AA)$, according to the data reported in Ref. 45. As can be noticed, $\mathrm{Ar}$ spectrum is broad band, however, most of the spectrally filtered radiation falls within the "water-window" spectral range. Completely different character of the spectral emission shows Fig. 2(b) for the nitrogen plasma. Nitrogen atoms are much lighter than argon atoms. They have also much less electrons, resulting in much narrower and less rich. Figure depicts nitrogen emission spectrum in the wavelength range from $\lambda=1.5 \mathrm{~nm}$ to $6 \mathrm{~nm}$. Measured spectrum is quasi-monochromatic, consisting of $\mathrm{N}^{5+}: 1 \mathrm{~s}^{2}-1 \mathrm{~s} 2 \mathrm{p}$ He-like nitrogen line at $\lambda=28.787 \AA$ and $\mathrm{N}^{6+}: 1 \mathrm{~s}-2 \mathrm{p} \mathrm{H}-$ like line at $\lambda=24.779 \AA-$ dashed line. An $\mathrm{L}-\alpha$ Ti edge at $\lambda \sim 2.7 \mathrm{~nm}$ (dashed-dotted line) allows to filter out $\mathrm{H}$-like line almost completely (inset) thus most energy of the beam, used later for illumination of the sample, resides in a single line at $\lambda=2.88 \mathrm{~nm}$ (solid line) in the SXR spectral range. Small peak at $\lambda=5.76 \mathrm{~nm}$ is attributed to the second order diffraction $(\mathrm{m}=2)$ of the He-like nitrogen line.

Additionally, other parameters, such as plasma size and photon flux were measured for both argon and nitrogen gas puff target sources. A spatial distribution of the plasmas in the "water-window" spectral range was obtained using a pinhole camera. The measurements were performed with a $\mathrm{Ti}$ filter, to assess the plasma size particularly in the "water-window" spectral range. Laser drilled, $32 \mu \mathrm{m}$ in diameter

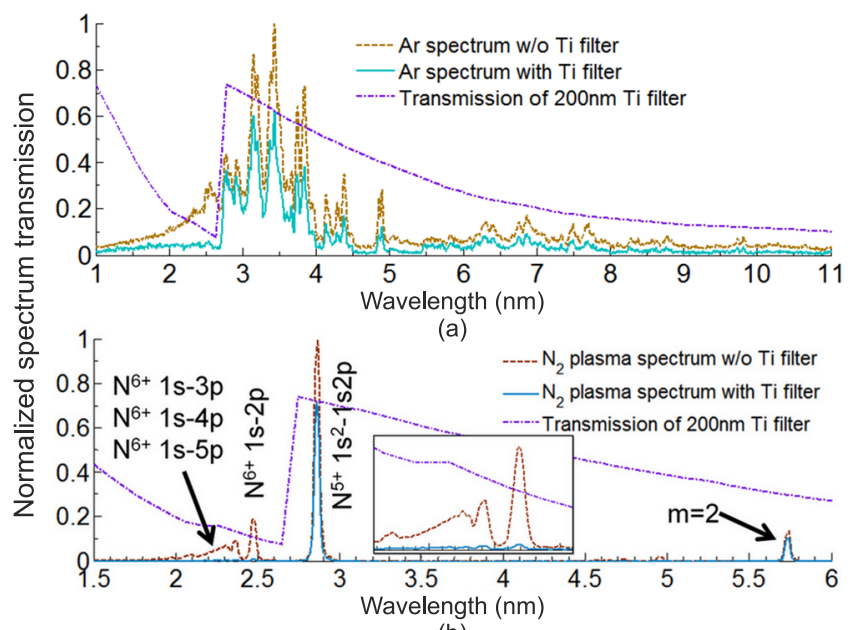

(b)

Fig. 2. Spectrum of the SXR radiation from the argon plasma (a) and from the nitrogen plasma (b), generated by focused Nd:YAG laser pulses irradiating a double stream gas puff target. pinhole was positioned almost half way between the plasma and the CCD camera (X-vision M25, Reflex s.r.o., Czech Republic), equipped with $512 \times 512$ pixels CCD chip, $0.5 \times 0.5 \mathrm{in}^{2}$ in size. The lateral magnification of the pinhole camera was 0.96x. The FWHM size of the argon plasma was $240 \times 130 \mu \mathrm{m}^{2}$. For the nitrogen plasma the magnification of the camera was $0.86 \mathrm{x}$ and measured FWHM size was slightly larger $-0.31 \times 0.47 \mathrm{~mm}^{2}$. Measurements of the source photon flux were performed using commercial AXUV100 silicon p-n junction photodiode, from International Radiation Detectors, Inc., and corrected for transmission of the $\mathrm{Ti}$ filter. The number of photons in the focal plane of the condenser (mentioned in the next section of the paper) for the argon plasma was equal to $3.6 \times 10^{10}$ photons/pulse, which corresponds to $2.3 \mu \mathrm{J} / \mathrm{pulse}$ in band. For the nitrogen plasma $3.86 \cdot 10^{9}$ photons/pulse was measured in the transmission band of the $200 \mathrm{~nm}$ thick Ti filter $(\lambda=2.8-6 \mathrm{~nm})$, which corresponds to energy of $256 \mathrm{~nJ} /$ pulse in band. More details about characterization and optimization of both argon and nitrogen target sources can be found in Ref. 43 and in Ref. 44, respectively.

\section{Achromatic SXR microscope}

The experimental setup of the achromatic SXR microscope is shown in Fig. 3. The microscope was equipped with an ellipsoidal, axi-symmetrical nickel coated SXR condenser, developed by Rigaku Innovative Technologies Europe Inc. It was used to focus the SXR radiation, emitted from the gas puff target source, onto an object. The condenser is a broad band optic, efficiently reflecting radiation from the EUV range down to the SXR region with a photon energy cut-off of $\sim 600 \mathrm{eV}$. The entrance diameter of the optic was equal to $14 \mathrm{~mm}$ and results in entrance numerical aperture of

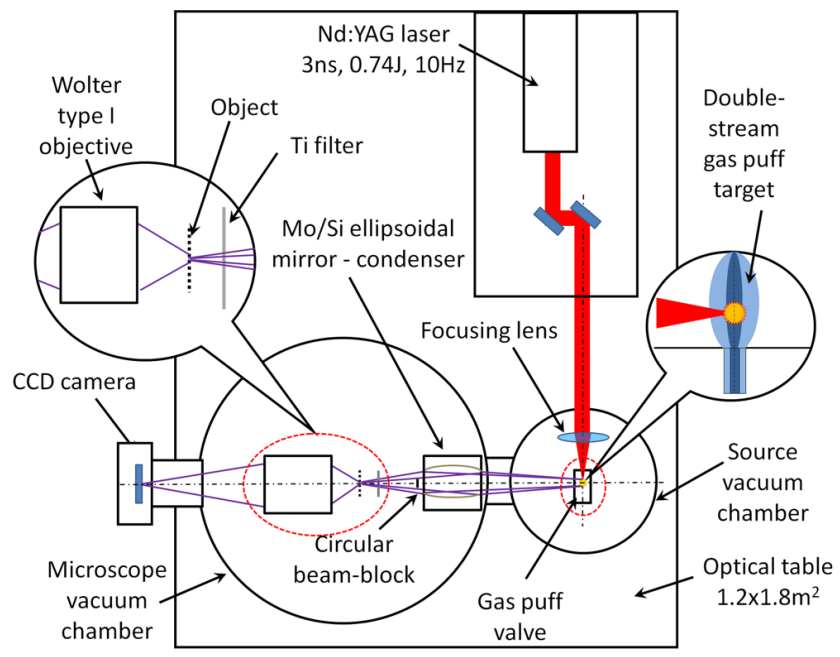

Fig. 3. Scheme of the achromatic SXR microscope, employing Wolter type-I objective. All the major components are indicated. 
$\mathrm{NA}_{\mathrm{C} \text { in }}=0.05$, while the exit diameter of $11.7 \mathrm{~mm}$ corresponds to exit numerical aperture of $\mathrm{NA}_{\mathrm{C}_{\text {_out }}}=0.09$. To spectrally narrow the emission from the argon plasma a $200 \mathrm{~nm}$ thick, free-standing titanium filter was used. Spectrally filtered radiation illuminates the sample, positioned in the second focal point of the condenser. For image formation Wolter type-I reflective objective is used [46]. The objective is composed of two axially-symmetric ellipsoidal and hyperboloidal nickel coated mirrors. The magnification of the objective is constant, defined by the curvature of both mirrors, and equal to 14.6x. The exit numerical aperture of the condenser $\left(\mathrm{NA}_{\mathrm{C}_{\text {_out }}}=0.09\right)$ is almost twice the objective entrance $\mathrm{NA}_{\mathrm{O}_{-} \text {in }}=0.05$, providing incoherent illumination [47], since $\sigma=\mathrm{NA}_{\mathrm{C}_{\text {_out }}} / \mathrm{NA}_{\mathrm{O}_{-} \text {in }}=1.8$. The magnified image of the object was formed onto an SXR-sensitive CCD camera (i-Kon, Andor) in transmission mode.

A test object - square pattern of copper mesh, Figure 4(a), was imaged with a half-pitch spatial resolution of $1.1+/-0.2 \mu \mathrm{m}$, measured using a well-known knife-edge (KE) criterion - Fig. 4(d). The knife-edge criterion states that for incoherent illumination 10-90 \% intensity transition across a sharp edge corresponds to a well-known Rayleigh resolution and to twice the value of half-pitch grating resolution of the optical system [48]. Typical image of the test object, depicted in Fig. 4(b), and real biological object, such
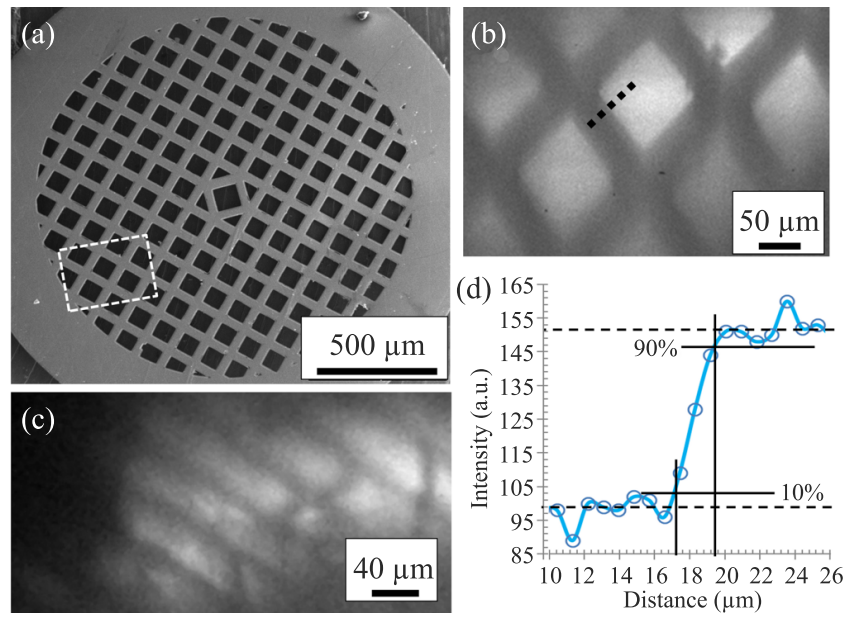

Fig. 4. SEM image of the test object - square mesh (a). The SXR image (b) of the same mesh, obtained in the region indicated in (a). SXR image of the biological sample - onion skin cells (c), showing high optical contrast between carbon-rich cell membranes and cells interior, which is highly transmissive to "water-window" radiation. KE resolution test result (d) showing normalized intensity profile across a sharp edge of the mesh. A 10-90\% intensity transition, corresponding to Rayleigh resolution, is equal to $2.2 \mu \mathrm{m}$, half-pitch spatial resolution equal to $1.1 \mu \mathrm{m}$, measured from the image of the mesh (b) in the region indicated in black dotted line. All data in images (b)-(d) were obtained using a broad band SXR emission from the argon plasma.
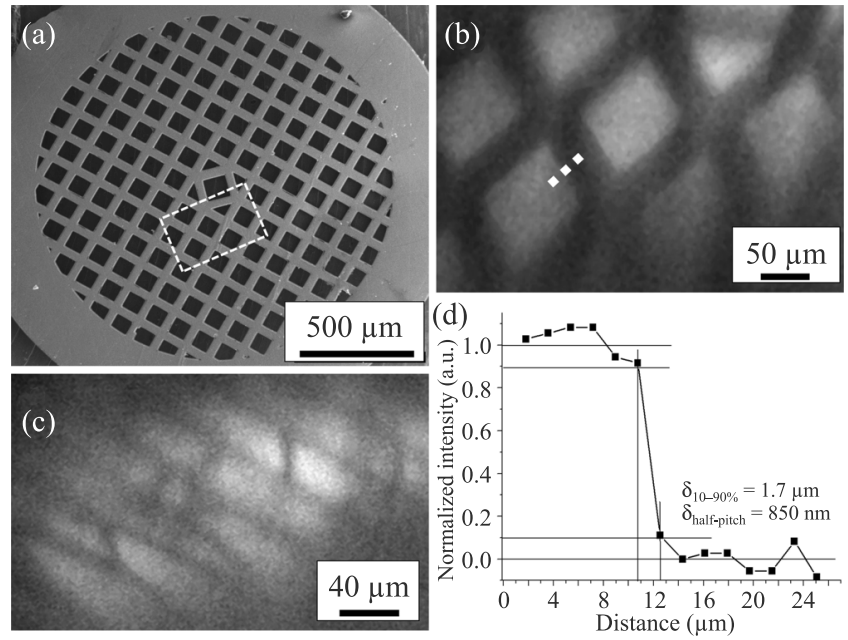

Fig. 5. Same as Fig. 4, but all data in images (b)-(d) were obtained using a narrow band, quasimonochromatic SXR emission from the nitrogen plasma. The knife edge measurement depicted in (d) was performed in

the in the region indicated in white dotted line in subimage (b).

as onion skin cells, in Fig. 4(c), obtained under broad band illumination from the argon plasma are shown [43]. To obtain a single image in the "water-window" spectral range 50-100 SXR pulses were necessary, at $10 \mathrm{~Hz}$ repetition rate. During image acquisition the CCD camera was cooled down to $-10^{\circ} \mathrm{C}$ to decrease the intrinsic, thermal noise of the detector. A pressure of $1.5 \times 10^{-4}$ mbar was constantly maintained in the microscope chamber during thesource operation.

Similar imaging experiments were performed using the nitrogen plasma illumination. As was already mentioned, nitrogen plasma emits quasimonochromatic radiation in the "water-window" spectral range. Due to this fact, the number of measured photons that can be used to produce an image is roughly an order of magnitude smaller than in case of a broad band illumination from the argon plasma. The same test object, presented in Fig. 5(a), was imaged with a slightly better spatial resolution of $0.85+/-0.12 \mu \mathrm{m}$, measured in the same way as before - Fig. 5(d). Typical SXR image of the mesh is depicted in Fig. 5(b), while, for comparison, image of the onion skin cells is presented in Fig. 5(c). The last figure depicts a few interconnected cells with dark cell walls and white cell interiors. Those images were obtained under narrow band illumination from the nitrogen plasma [44]. Dashed box in the SEM images in Figs. 4(a) and Fig. 5(a) indicate regions of the sample imaged with the SXR microscope. The advantage of the "water-window" optical contrast near the oxygen-edge is obvious, since the onion skin sample thickness in this case is $\sim 40 \mu \mathrm{m}$, which makes it completely opaque in the EUV spectral range for example.

The obtained spatial resolution is limited in this system mostly by a point spread function (PSF) of the Wolter optics. It is due to the fact that it is not a full aperture optics, but has 
a central beam block, forming a thin, annular shaped entrance aperture, with an obscuration ratio of 0.93 . Such entrance aperture will result in slightly narrower first lobe of the PSF, however, the higher order lobes will be much more pronounced, causing knife edge function (KEF) to be much wider. Additional resolution limiting factors are the modulation transfer function of the CCD camera, mirror surface curvature errors, resulting in various aberrations of Wolter objective, optical quality of its mirrors, and, finally, surface roughness of the two-mirror optical system. More details about presented imaging experiments for both argon and nitrogen plasma illumination can be found in Ref. 43 and in Ref. 44, respectively.

Nevertheless, the main advantage of the achromatic SXR microscopy system is the lack of dispersion of the condenser and objective, which makes possible to change spectral emission of the source. It can be achieved by changing either the working gas or the filters, and allows to perform imaging in other wavelength ranges, for example $18-60 \mathrm{~nm}$ using $\mathrm{Al}$ filter. This opens new possibilities to exploit different spectral information, now possible to obtain from investigated objects at various wavelengths and allow in turn studying the samples more thoroughly. The other advantage is the fact that the experimental setup of such microscope is extremely compact. The microscope is located inside a vacuum chamber, $60 \mathrm{~cm}$ in diameter and $35 \mathrm{~cm}$ in height and the entire system fits on top of a single $1.8 \times 1.2 \mathrm{~m}^{2}$ optical table.

\section{Nanometer spatial resolution SXR microscope}

In this section a further development of presented microscopy systems will be discussed. The resolution of those systems may be unsatisfactory for many applications that require a nanometer spatial resolution, thus, to improve the spatial resolution, a new system was developed. It employs previously described laser-plasma source, based on a double nitrogen/helium gas puff target, emitting a quasi-monochromatic, incoherent radiation, in the "water-widow" spectral range at $\lambda=2.88 \mathrm{~nm}$. The source was optimized particularly for efficient SXR generation with measured number of photons in the focal plane of the condenser to be $7.9 \cdot 10^{9}$ photons/pulse, which corresponds to energy of 561 $\mathrm{nJ} /$ pulse. The source was coupled to an ellipsoidal, grazing incidence condenser and a Fresnel zone plate objective. Wavelengths near K- $\alpha$ oxygen edge are preferable for biological imaging, because of highest transmission and largest absorption length in water $\sim 10 \mu \mathrm{m}$, which allows to image thicker samples. Additionally, quasi-monochromatic emission, corresponding to radiative transitions in helium-like nitrogen ions, is suitable for highly dispersive Fresnel zone plate objective.

The experimental setup of the nanometer resolution SXR microscope is shown in Fig. 6. The SXR radiation from the

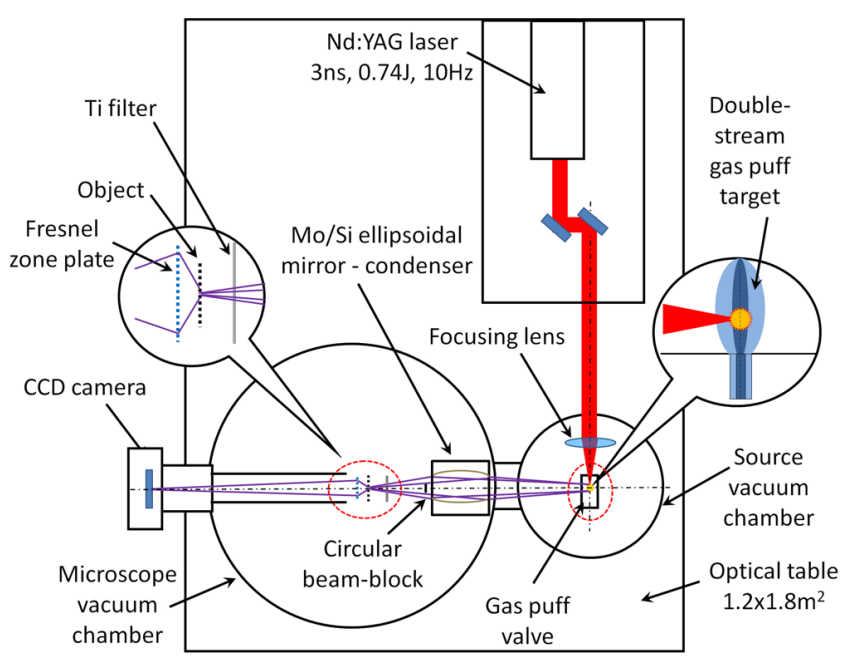

Fig. 6. Scheme of the nanometer spatial resolution SXR microscope, employing a Fresnel zone plate objective. All the major components are indicated.

nitrogen plasma is collected and focused by an ellipsoidal, axi-symmetrical nickel coated condenser mirror (Rigaku Innovative Technologies Europe s.r.o.), as before, however the condenser in this system was reversed to match its numerical aperture to the numerical aperture of the objective Fresnel zone plate optic. For this reason the entrance and exit diameters of the condenser are now equal to $11.7 \mathrm{~mm}$ and $14 \mathrm{~mm}$, which results in the entrance numerical aperture of NAC_in $=0.09$ and the exit numerical aperture of NAC_out $=0.05$, respectively. The previously described Wolter objective was equipped with a large circular aperture to block a stray light along the optical axis. In this system an auxiliary beam block, $3.25 \mathrm{~mm}$ in diameter, was suspended on two crossed copper wires downstream the condenser. A $200 \mathrm{~nm}$ thick Ti filter was employed as well, for efficient attenuation of the hydrogen-like nitrogen emission line at $2.48 \mathrm{~nm}$ wavelength, which turned out to be below the L- $\alpha$ Ti edge at $\lambda \sim 2.7 \mathrm{~nm}$ (dashed-dotted line in Fig. 2(b). Filtered SXR radiation (helium like nitrogen line at $2.88 \mathrm{~nm}$ wavelength) illuminates the sample, which is then imaged using a Fresnel zone plate objective onto an SXR sensitive back-illuminated CCD camera. Two CCD cameras were used: $2048 \times 2048$ pixels, $13 \times 13 \mu \mathrm{m}^{2}$ pixel size, GE $20482048 \mathrm{BI}$, from Greateyes $\mathrm{GmbH}$., and previously used Andor i-Kon CCD camera, $1024 \times 1024$ pixels, $13 \times 13 \mu \mathrm{m}^{2}$ pixel size. The zone plate (Zoneplates Ltd., UK), $250 \mu \mathrm{m}$ in diameter has the outer zone width of $30 \mathrm{~nm}$ and the focal length $\mathrm{f}=2.6 \mathrm{~mm}$ at $2.88 \mathrm{~nm}$ wavelength. As can be noticed, the numerical aperture of the zone plate, $\mathrm{NA}_{\mathrm{ZP}}=0.048$, is matched to the numerical aperture of the condenser $\mathrm{NA}_{\mathrm{C}_{\text {_out }}}$, providing incoherent illumination, [47], since $\sigma=$ NAC_out $/ \mathrm{NA}_{\mathrm{ZP}}=\sim 1$. The lowest geometrical magnification of the system (object 
to CCD chip) is $\sim 220 x$. Typically, image on the computer screen is $\sim 10 \mathrm{x}$ larger than the CCD chip (default image size in the Andor software for 19" monitor), thus the lowest total image magnification is above 2200x. More details about the system development and characterization can be found in Ref. 49. The system was also thoroughly characterized in terms of a signal to noise ratio (SNR), providing important clues for optimal image acquisition in terms of the exposure, CCD detector temperature, readout speed and chip spatial integration (Eng. binning). The optimization allowed achieving highest SNR and the possibility of "single shot" imaging. Such approach allowed for one SXR pulse, 3-4 ns in duration, to be used for image acquisition, resulting in a nanosecond temporal resolution imaging [43,44].

As a test object a copper, 2000 periods per inch TEM mesh from SPI supplies, USA, was used. It has $7.5 \mu \mathrm{m}$ squared holes and $5 \mu \mathrm{m}$ width bars, much smaller comparing to mesh used in the previous experiments (period $124 \mu \mathrm{m}$ and bar width $83 \mu \mathrm{m}$ ). Image of the mesh, obtained using GE 20482048 BI camera, is depicted in Fig. 7(a). Structure of the mesh is clearly visible. To acquire this image, 100 SXR pulses were accumulated over 10 seconds exposure time. Field of view (FOV) is equal to $120 \times 120 \mu \mathrm{m}^{2}$. Some imperfections, magnified in the inset images, are also visible, including $200 \mathrm{~nm}$ wide feature, located inside one of the square holes in the mesh. Resolution of the microscope was measured again, employing a well-established knife edge test. A typical KE lineout, obtained at location indicated with white line, is depicted in Fig. 7(b), where 10-90\% intensity transition in the normalized lineout is $\sim 120 \mathrm{~nm}$ (Rayleigh resolution), thus, half-pitch spatial resolution is equal to $60 \mathrm{~nm}$.
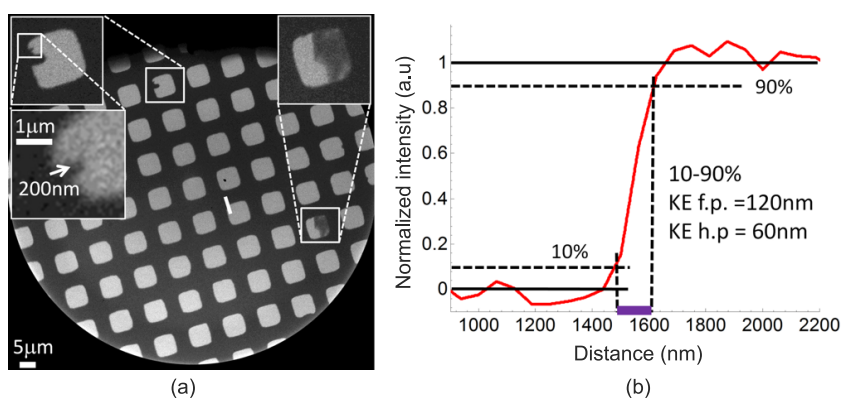

(b)

Fig. 7. The SXR image of the copper \#2000 mesh obtained in the "water-window" spectral range (a). Exposure equals to 100 SXR pulses $/ 10$ seconds, field of view $-120 \times 120 \mu \mathrm{m}^{2}$. Inset images show some imperfections in the mesh, including $200 \mathrm{~nm}$ wide feature. $\mathrm{KE}$ resolution test result (b) showing normalized intensity profile across a sharp edge. A 10-90\% intensity transition, corresponding to Rayleigh resolution, is equal to $120 \mathrm{~nm}$, half-pitch spatial resolution equal to $60 \mathrm{~nm}$, measured from the image of the mesh (a). The lineout was obtained at the location indicated with white line.

\subsection{Applications of the nanometer spatial resolution SXR microscope}

Some examples of "water-window" images of various objects are depicted in Fig. 8 showing applicability of such system to various fields of science and technology. The images were acquired with i-Kon camera with FOV of $60 \times 60 \mu \mathrm{m}^{2}$. Image of the test object (mesh) obtained with single, 3-4 ns in duration, SXR pulse is depicted in Fig. 8(a). The signal from a $4 \times 4$ matrix of pixels of the CCD chip $(4 \times 4$ binning) was integrated to improve the SNR. The spatial resolution was estimated to be $\sim 240 \mathrm{~nm}$ (half-pitch). A carbon membrane, $15 \mathrm{~nm}$ thick with $1.2 \mu \mathrm{m}$ in diameter holes was imaged, together with the supporting structure, as depicted in Fig. 8(b). The carbon membrane was additionally coated with $60 \mathrm{~nm}$ of gold in a standard evaporator to decrease the transmittance of the membrane, from $93.6 \%$ down to $18.4 \%$, according to the CXRO data [45]. The image shows semitransparent membrane with the holes in top-right and bottom-left part of the image. To acquire this image, 50 SXR pulses were accumulated over 5 seconds exposure time.

A silicon nitride membrane, $50 \mathrm{~nm}$ thick, was used as a support for a thin layer of saccharose $\left(\mathrm{C}_{12} \mathrm{H}_{22} \mathrm{O}_{11}\right)$, as depicter in Fig. 8(c). It was possible to measure the transmittance of a single $(71.8 \%)$ and double $(52.3 \%)$ saccharose layers. From that the layer thickness of $\sim 160 \mathrm{~nm}$ was calculated. This image required 200 SXR pulses, 20 seconds exposure. A supercoiled pBR322 plasmid DNA (4361bp), circular double-stranded DNA from Inspiralis, UK, was deposited on top of $50 \mathrm{~nm}$ thick $\mathrm{Si}_{3} \mathrm{~N}_{4}$ membrane, from $100 \mathrm{ng} / \mu \mathrm{l}$ solution. Subsequently, it was dried in nitrogen atmosphere for 20 minutes. The image of aggregations of DNA strands, obtained in the ,water window” spectral range, is depicted in Fig. 8(d). The aggregations are clearly visible; some of them are separated from the "DNA bulk" and vary in size from a few microns down to hundreds of nanometers. To acquire this image, 500 SXR pulses were accumulated - 50 seconds exposure time. More information about those measurements is presented in Ref. 49.

Another example is SXR imaging of gold microcracks. A $100 \mathrm{~nm}$ thick silicon nitride membrane was coated with a $100 \mathrm{~nm}$ thick gold layer, deposited by evaporation, which caused some microcracks to develop in the gold layer. That was observed using a visible light microscope with NA $=0.7$ and 40x magnification objective - Fig. 8(e). For comparison, the SXR image was acquired with the SXR microscope, Fig. 8(f). Estimated gold layer SXR transmittance is $\sim 6.6 \%$ at $2.88 \mathrm{~nm}$ wavelength radiation, based on the CXRO data [52]. A high resolution image was obtained with features visible beyond the diffraction limit of the ordinary visible light microscope $-0.61 \lambda / \mathrm{NA} \sim 480 \mathrm{~nm}$. The SXR image was obtained with 500 SXR pulses at $10 \mathrm{~Hz}$ source repetition rate. Arrow length (for a scale) equals to $18.8 \mu \mathrm{m}$. The 

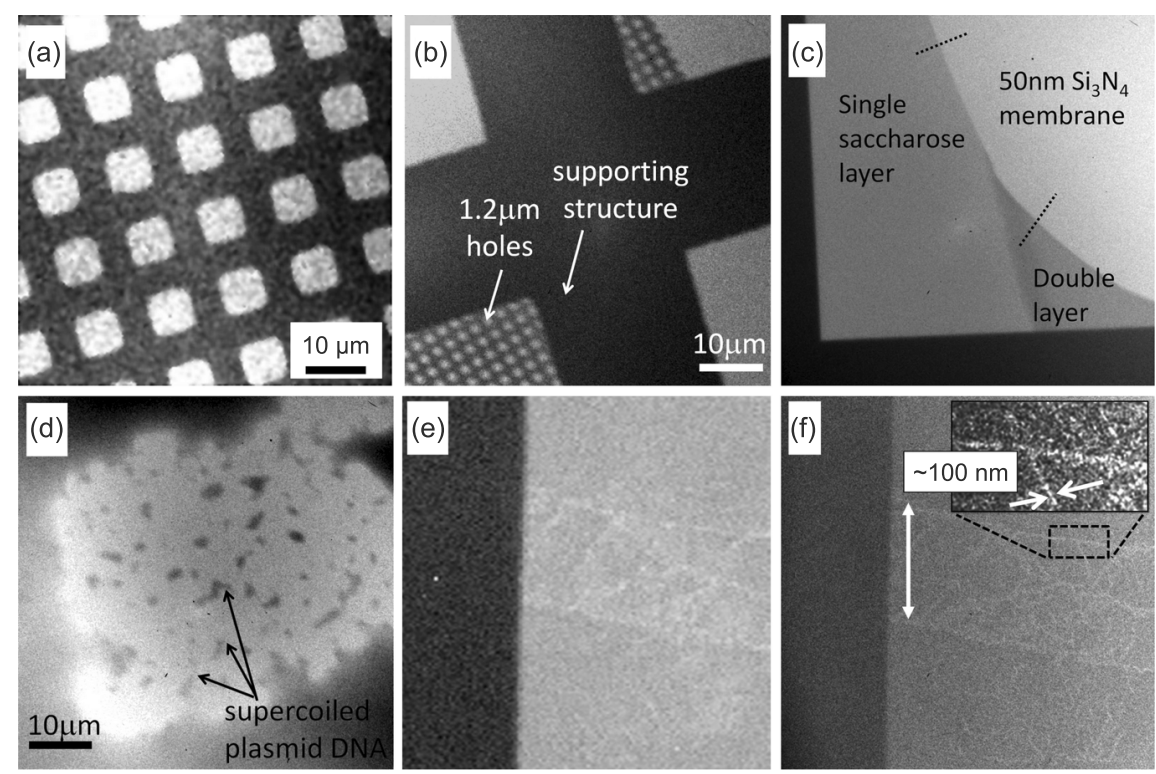

Fig. 8. SXR image of the copper mesh (a), obtained with a single, 3-4 ns SXR pulse, with spatial resolution (half-pitch) of $240 \mathrm{~nm}$. Image was obtained by binning a signal from a $4 \times 4$ matrix of CCD pixels. Examples of "water-window" images of various objects obtained using the SXR microscope. A $15 \mathrm{~nm}$ thick carbon membrane, coated additionally with $60 \mathrm{~nm}$ of gold, having an array of $1.2 \mu \mathrm{m}$ in diameter holes (b). The membrane is supported by a mesh-like structure. Acquisition parameters: 50 SXR pulses, 5 seconds exposure time. A $50 \mathrm{~nm}$ thick silicon nitride membrane coated with $\sim 160 \mathrm{~nm}$ thick layer of saccharose (c). Acquisition parameters: $200 \mathrm{SXR}$ pulses, 20 seconds exposure time. A supercoiled pBR322 plasmid DNA, deposited on top of $50 \mathrm{~nm}$ thick silicon nitride membrane, from $100 \mathrm{ng} / \mu \mathrm{l}$ solution (d). Acquisition parameters: 500 SXR pulses, 50 seconds exposure time. A $100 \mathrm{~nm}$ thick silicon nitride membrane with $100 \mathrm{~nm}$ thick gold layer with microcracks observed using a visible light microscope ( $\mathrm{NA}=0.7,40 \mathrm{x}$ objective) $-(\mathrm{e})$ and the SXR microscope (f). The SXR image was obtained with 500 SXR pulses at $10 \mathrm{~Hz}$ repetition rate, detector temperature $\mathrm{T}=-20^{\circ} \mathrm{C}$, and the $\mathrm{CCD}$ readout rate $-2.5 \mathrm{MHz}$. Arrow length equals to $18.8 \mu \mathrm{m}$. Small, contrast-enhanced inset in Fig. (f) shows a branched microcrack in more detail. The width of the crack was $\sim 100 \mathrm{~nm}$. All images FOV is $60 \times 60 \mu \mathrm{m}^{2}$.

cracks, due to the diffraction limit for visible light microscope, appear in Fig. 8(e) as barely visible and have approximately $500 \mathrm{~nm}$ in width. A small, contrast-enhanced inset in Fig. 8(f), shows a branched crack in more detail due to higher spatial resolution achievable with the SXR radiation. The width of the crack was $\sim 100 \mathrm{~nm}$. The microcrack sample was prepared through collaboration with Czech Technical University in Prague.
A biological sample was also investigated and the results are presented in Fig. 9. The biological specimen was prepared from dehydrated adherent CT 26 fibroblasts, derived from colon carcinoma Mus musculus (strain BALB/c). The cells were deposited on top of $100 \mathrm{~nm}$ thick $1 \times 1 \mathrm{~mm}^{2} \mathrm{Si}_{3} \mathrm{~N}_{4}$ membrane. The visible light microscopy image, Fig. 9(a), is directly compared to the SXR image of the cells, depicted in Fig. 9(b). The cell size is approximately $20-30 \mu \mathrm{m}$. The

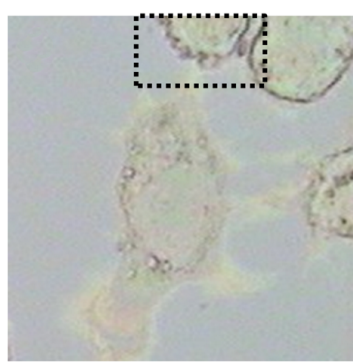

(a)

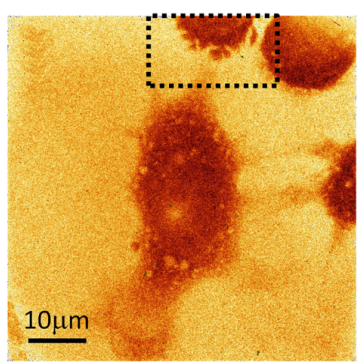

(b)

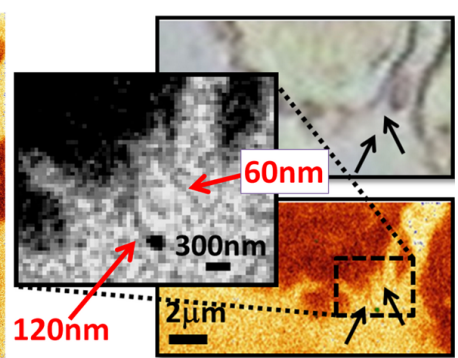

(c)

Fig. 9. Visible light microscopy image (a) and, for comparison, the SXR image (b) of dehydrated CT 26 fibroblasts, derived from colon carcinoma Mus musculus (strain BALB/c) placed on top of $100 \mathrm{~nm}$ thick silicon nitride membrane. The images show cells with internal structure visible due to a phase contrast in visible light microscope image and as modulation in the transmittance of the SXR light through the sample in the SXR image. Image (b) is presented in false colors. Image (c) shows direct comparison of small areas and smallest features in the visible light microscopy image and the SXR image. The SXR image show enhanced spatial resolution and high absorption contrast. 
Recent advancements in the "water-window" microscopy with laser-plasma SXR source based on a double stream...

internal structures of the cells are visible due to a phase contrast in visible light microscope image and as a modulation in the transmittance of the $2.88 \mathrm{~nm}$ wavelength light through the sample in the SXR image. The SXR image was acquired with 600 SXR pulses -1 minute exposure, detector temperature $-20^{\circ} \mathrm{C}$, and the $\mathrm{CCD}$ readout speed $-50 \mathrm{kHz}$. The visible light microscopy image was obtained with 40x objective and NA $=0.7$. Fig. 9(c) shows a direct comparison of small areas, indicated with black dotted boxes in the visible light microscopy image and the SXR image, in Figs. 9(a) and (b). The SXR image shows enhanced spatial resolution due to much shorter wavelength, beyond the capabilities of diffraction limited visible light microscopes. The internal and external structures of the cells, of the order of $\sim 60 \mathrm{~nm}$, are clearly visible in the SXR image, as indicated with arrows in Fig. 9(c); while in the visible light micrographs cannot be resolved. The carcinoma cell sample was prepared through collaboration with Czech Technical University in Prague. More details about this work can be found in Ref. 53.

\section{Conclusions}

In this short overview paper I have presented recent achievements and advancements in the development of compact, table-top laser-plasma double stream gas puff target based "water-window" microscopes. Two different constructions were shown and discussed. The first one was an achromatic SXR microscope. It was equipped with Wolter type-I objective, which produced a similar quality and resolution images of objects with broad band emission from the argon plasma and narrowband emission from the nitrogen plasma. This system allows capturing magnified images of the objects, with magnification of $\sim 15 \mathrm{x}, \sim 1 \mu \mathrm{m}$ (or less) half-pitch spatial resolution and exposure time as low as 5 seconds. A detailed characterization and optimization of both: the source and the microscope setup were presented. Although, the resolution of such achromatic system was quite low for many applications, the system offers imaging capability in the "water-window" spectral range, combining its desk-top size, accessibility and simple operation. Most importantly, however, due to lack of dispersion of the condenser and objective such achromatic SXR microscope makes possible to exploit different spectral information about the object. Such new information is now possible to obtain from investigated objects at various wavelengths. This can be done by changing the spectral emission of the source and allows, in turn, studying the samples more thoroughly.

Nevertheless, for many applications a micron spatial resolution may not be sufficient, especially for nanotechnology, where the spatial resolution should be of the order of less than $100 \mathrm{~nm}$. To overcome this limitation a second system was developed. Such "water-window" compact, desk-top microscope, was based on nitrogen double stream gas puff target SXR source and Fresnel zone plate objective. The microscope allowed capturing magnified images of the objects, with $60 \mathrm{~nm}$ half-pitch spatial resolution, magnification of 220x or higher, exposure time as low as a few seconds, desk-top footprint, easy accessibility and simple operation. Such system offers superior spatial resolution to the visible light microscopes, does not require sample preparation like SEM microscopes, exploits natural, optical contrast in the "water-window" spectral range for biological imaging applications and may be considered as a complementary imaging tool to the already well established techniques. It was also shown that this compact SXR high resolution microscope is capable of imaging variety of different samples from various fields of science and technology, including, but not limited to, biology, material science and nanotechnology. Such microscope brings together very important factors, such as the desk-top size, fast exposures and nanometer spatial resolution, the key parameters opening possibility of widespread and commercialization of such systems in the near future.

\section{Acknowledgements}

This work was supported by the National Science Centre; award numbers UMO-2015/17/B/ST7/03718, UMO-2015/19/ B/ST3/00435 and the National Centre for Research and Development, LIDER programme; award \# LIDER/004/410/L-4/ 12/NCBR/2013 and the 7th Framework Programme's Laserlab Europe III project (No. 284464). This work has received funding also from the European Union's Horizon 2020 research and innovation program under grant agreement No. 654148 Laserlab Europe IV.

I would like to express my gratitude to Alfio Torrisi, Dr. Andrzej Bartnik, Marcin Skorupka, Dr. Jerzy Kostecki, Dr. Roman Jarocki, Dr. Mirosław Szczurek, Lukasz Wegrzynski, Tomasz Fok, Prof. Henryk Fiedorowicz, Muhammad Fahad Nawaz, Daniel Adjei, Šárka Vondrová, Jana Turňová, Dr. Alexandr Jančarek, Prof. Jiří Limpouch and Prof. Miroslava Vrbová for their contributions to the original work presented in mentioned publications. Additionally, I express my gratitude to Dr. Martin Regehly, from greateyes GmbH., Germany, for the possibility to use their GE 20482048 BI CCD camera. I thank Prof. L. Pina for his help in the preparation of SXR condenser optics and to Prof. René Hudec for Wolter objective used in the original work.

\section{References}

1. L.B. Da Silva, J.E. Trebes, R. Balhorn, S. Mrowka, E. Anderson, D.T. Attwood, T.W. Barbee Jr., J. Brase, M. Corzett, J. Gray, J.A. Koch, C. Lee, D. Kern, R.A. London, B.J. MacGowan, and D.L. Mathews, "X-ray Laser Microscopy with an Rat Sperm Nuclei”, Science 258, 269 (1992).

2. D.S. DiCicco, D. Kim, R. Rosser, and S. Suckewer, "First stage in the development of a soft-x-ray reflection imaging micro- 
scope in the Schwarzschild configuration using a soft-x-ray laser at 18.2 nm", Opt. Lett. 17, 2, 157-159 (1992).

3. L.B. Da Silva, J.E. Trebes, S. Mrowka, T.W. Barbee, Jr., J. Brase, J.A. Koch, R.A. London, B.J. MacGowan, D.L. Matthews, D. Minyard, G. Stone, T. Yorkey, E. Anderson, D.T. Attwood, and D. Kern, "Demonstration of X-ray microscopy with an x-ray laser operating near the carbon K edge", Opt. Lett. 17, 10, 754-756 (1992).

4. M. Wieland, C. Spielmann, U. Kleineberg, T. Westerwalbesloh, U. Heinzmann, and T. Wilhein, "Toward time-resolved soft X-ray microscopy using pulsed fs-high-harmonic radiation", Ultramicroscopy 102, 93 (2005).

5. M. Kishimoto, M. Tanaka, R. Tai, K. Sukegawa, M. Kado, N. Hasegawa, H. Tang, T. Kawachi, P. Lu, K. Nagashima, H. Daido, Y. Kato, K. Nagai, and H. Takenaka, "Development of soft X-ray microscopy System using X-ray laser in JAERI Kansai”, Journal de Physique IV 104, 141-144 (2003).

6. I.A. Artioukov, A.V. Vinogradov, V.E. Asadchikov, Y.S. Kasyanov, R.V. Serov, A.I. Fedorenko, V.V. Kondratenko, and S.A. Yulin, "Schwarzschild soft-x-ray microscope for imaging of nonradiating objects", Opt. Lett. 20, 2451-2453 (1995).

7. G. Vaschenko, F. Brizuela, C. Brewer, M. Grisham, H. Mancini, C.S. Menoni, M.C. Marconi, J.J. Rocca, W. Chao, J.A. Liddle, E.H. Anderson, D.T. Attwood, A.V. Vinogradov, I.A. Artioukov, Y.P. Pershyn, and V.V. Kondratenko, "Nanoimaging with a compact extreme-ultraviolet laser", Opt. Lett. 30, 16, 2095 (2005)

8. C.A. Brewer, F. Brizuela, P. Wachulak, D.H. Martz, W. Chao, E.H. Anderson, D.T. Attwood, A.V. Vinogradov, I.A. Artyukov, A.G. Ponomareko, V.V. Kondratenko, M.C. Marconi, J.J. Rocca, and C.S. Menoni, "Single-shot extreme ultraviolet laser imaging of nanostructures with wavelength resolution". Opt. Lett. 33, 518, (2008), this article was also published in Virtual Journal for Biomedical Optics, 3, 4, (2008) and in Virtual Journal of Nanoscale Science \& Technology, 17, 15, (2008).

9. P.W. Wachulak, C.A. Brewer, F. Brizuela, W. Chao, E. Anderson, R.A. Bartels, C.S. Menoni, J.J. Rocca, and M.C. Marconi, "Analysis of extreme ultraviolet microscopy images of patterned nanostructures based on a correlation method", Journal of the Optical Society of America B, 25, B20, (2008), this article was also published in The Virtual Journal for Biomedical Optics, 3, 8, (2008).

10. G. Vaschenko, C. Brewer, F. Brizuela, Y. Wang, M.A. Larotonda, B.M. Luther, M.C. Marconi, J.J. Rocca, and C.S. Menoni, "Sub-38 nm resolution tabletop microscopy with 13 nm wavelength laser light", Opt. Lett. 31, 9, 1214 (2006).

11. P.W. Wachulak, A. Bartnik, H. Fiedorowicz, T. Feigl, R. Jarocki, J. Kostecki, R. Rakowski, P. Rudawski, M. Sawicka, M. Szczurek, A. Szczurek, and Z. Zawadzki, „A compact, quasi-monochromatic laser-plasma EUV source based on a double-stream gas-puff target at $13.8 \mathrm{~nm}$ wavelength", Appl. Phys. B 100, 3, 461-469, (2010).

12. P.W. Wachulak, A. Bartnik, and H. Fiedorowicz, "Sub-70 nm resolution tabletop microscopy at $13.8 \mathrm{~nm}$ using a compact laser-plasma EUV source", Opt. Lett. 35, 14, 2337-2339 (2010).
13. P.W. Wachulak, A. Bartnik, H. Fiedorowicz, and J. Kostecki, "A 50nm spatial resolution EUV imaging-resolution dependence on object thickness and illumination bandwidth", Opt. Express 19, 10, 9541-9550 (2011).

14. K.W. Kim, Y. Kwon, K.Y. Nam, J.H. Lim, K.G. Kim, K.S. Chon, B.H. Kim, D.E. Kim, J.G. Kim, B.N. Ahn, H.J. Shin, S. Rah, K.H. Kim, J.S. Chae, D.G. Gweon, D.W. Kang, S.H. Kang, J.Y. Min, K.S. Choi, S.E. Yoon, E.A. Kim, Y. Namba, and K.H. Yoon, "Compact soft $\mathrm{x}$-ray transmission microscopy with sub-50 nm spatial resolution", Physics in Medicine and Biology 51, N99-N107 (2006).

15. M. Berglund, L. Rymell, M. Peuker, T. Wilhein, and H.M. Hertz, "Compact water-window transmission X-ray microscopy", Journal of Microscopy 197, 3, 268-273 (2000).

16. G.A. Johansson, A. Holmberg, H.M. Hertz, and M. Berglund, "Design and performance of a laser-plasma-based compact soft x-ray microscope", Review of Scientific Instruments 73, 3, 1193 (2002).

17. M. Benk, K. Bergmann, D. Schäfer, and T. Wilhein, "Compact soft x-ray microscope using a gas-discharge light source", Opt. Lett. 33, 20, 2359 (2008).

18. P.A.C. Takman, H. Stollberg, G.A. Johansson, A. Holmberg, M. Lindblom \& H.M. Hertz, "High-resolution compact X-ray microscopy", Journal of Microscopy 226, 2, 175-181 (2007).

19. H. Legall, G. Blobel, H. Stiel, W. Sandner, C. Seim, P. Takman, D.H. Martz, M. Selin, U. Vogt, H.M. Hertz, D. Esser, H. Sipma, J. Luttmann, M. Hofer, H.D. Hoffmann, S. Yulin, T. Feigl, S. Rehbein, P. Guttmann, G. Schneider, U. Wiesemann, M. Wirtz, and W. Diete, "Compact X-ray microscope for the water window based on a high brightness laser plasma source", Opt. Express 20, 16, 18362 (2012).

20. D.H. Martz, M. Selin, O. von Hofsten, E. Fogelqvist, A. Holmberg, U. Vogt, H. Legall, G. Blobel, C. Seim, H. Stiel, and H.M. Hertz, "High average brightness water window source for short-exposure cryomicroscopy", Opt. Lett. 37, 21, 4425 (2012).

21. H. Legall, H. Stiel, G. Blobel, C. Seim, J. Baumann, S. Yulin, D. Esser, M. Hofer, U. Wiesemann, M. Wirtz, G. Schneider, and H.M. Hertz, "A compact Laboratory Transmission X-ray Microscope for the water window", J. Phys.: Conf. Ser. 463 012013 (2013).

22. M. Bertilson, O. von Hofsten, U. Vogt, A. Holmberg, and H.M. Hertz, "High-resolution computed tomography with a compact soft x-ray microscope", Opt. Express 17, 13, 11057 (2009).

23. K. Murakami, T. Oshino, H. Nakamura, M. Ohtani, and H. Nagata, "Schwarzschild microscope for carbon $\mathrm{K} \alpha$ radiation", Appl. Opt. 32, 34, 7057-7061 (1993).

24. W. Chao, J. Kim, S. Rekawa, P. Fischer, and E.H. Anderson, "Demonstration of $12 \mathrm{~nm}$ Resolution Fresnel Zone Plate Lens based Soft X-ray Microscopy", Opt. Express 17, 20, 17669 (2009).

25. S. Rehbein, S. Heim, P. Guttmann, S. Werner, and G. Schneider, "Ultrahigh-Resolution Soft-X-Ray Microscopy with Zone Plates in High Orders of Diffraction", Physical Review Letters 103, 110801 (2009).

26. B.L. Mesler, P. Fischer, W. Chao, E.H. Anderson, and D.H. Kim, "Soft X-ray imaging of spin dynamics at high spatial 
Recent advancements in the "water-window" microscopy with laser-plasma SXR source based on a double stream...

and temporal resolution”, J. Vac. Sci. Technol. B 25, 2598-2602 (2007).

27. P. Fischer, D. H. Kim, B.L. Mesler, W. Chao, E.H. Anderson, "Magnetic soft X-ray microscopy: Imaging spin dynamics at the nanoscale", Journal of Magnetism and Magnetic Materials 310, 2, 2689-2692 (2007).

28. P. Fischer, D.H. Kim, W. Chao, J.A. Liddle, E.H. Anderson, and D.T. Attwood, "Soft X-ray microscopy of nanomagnetism”, Materials Today 9, 1-2, 26-33 (2006).

29. D.H. Kim, P. Fischer, W. Chao, E. Anderson, M.Y. Im, S.Ch. Shin, and S.B. Choe, "Magnetic soft X-ray microscopy at 15 $\mathrm{nm}$ resolution probing nanoscale local magnetic hysteresis", J. Appl. Phys. 99, 08H303 (2006).

30. B. Nieman, D. Rudolph, and G. Schmahl, "Soft X-ray imaging zone plates with large zone numbers for microscopic and spectroscopic applications", Opt. Commun. 12, 160-163 (1974).

31. B. Nieman, D. Rudolph, G. Schmahl, "X-ray microscopy with synchrotron radiation", Appl. Opt. 15, 1883-1884 (1976).

32. G. Schneider, B. Riemann, P. Guttmann, D. Rudolph, G. Schmahl, "Cryo X-ray Microscopy”, Synchr. Rad. News 8, 19 (1995).

33. C. Jacobsen, S. Williams, E. Anderson, M.T. Browne, C.J. Buckley, D. Kern, J. Kirz, M. Rivers, and X. Zhang, "Diffraction-Limited Imaging in a Scanning Transmission X-ray Microscope", Opt. Commun. 86, 351 (1991).

34. C. Jacobsen, J. Kirz, S. Williams, "Resolution in soft X-ray microscopes", Ultramicroscopy 47, 55-79 (1992).

35. J. Kirz, C. Jacobsen, S. Lindaas, S. Williams, X. Zhang, E. Anderson, M. Howells, Soft X-ray Microscopy at the National Synchrotron Light Source, published in Synchrotron Radiation in the Biosciences, (Oxford Univ. Press, 1994), pp. 563.

36. G. Poletti, F. Orsini, D. Batani, "Study of Multicellular Living Organisms by SXCM (Soft X-Ray Contact Microscopy)", Solid State Phenomena 107, 7-10 (2005).

37. A.C. Cefalas, P. Argitis, Z. Kollia, E. Sarantopoulou, T.W. Ford, A.D. Stead, A. Marranca, C.N. Danson, J. Knott, and D. Neely, Technical Report RAL-TR-98-007, TMR Large-Scale Facilities Access Programme, National Hellenic Research Foundation, Greece (1998).

38. G. Schneider, "Cryo X-ray microscopy with high spatial resolution in amplitude and phase contrast", Ultramicroscopy $\mathbf{7 5}$, 85-104 (1998).

39. W. Meyer-Ilse, D. Hamamoto, A. Nair, S.A. Leličvre, G. Denbeaux, L. Johnson, A.L. Pearson, D. Yager, M.A. Legros, and C.A. Larabell, "High resolution protein localization using soft X-ray microscopy", Journal of Microscopy 201, 3, 395-403 (2001).

40. J. Maser, A. Osanna, Y. Wang, C. Jacobsen, J. Kirz, S. Spector, B. Winn \& D. Tennant, "Soft X-ray microscopy with a cryo scanning transmission X-ray microscope: I. Instrumentation, imaging and spectroscopy", Journal of Microscopy 197, 1, 68-79 (2000).
41. H. Fiedorowicz, A. Bartnik, R. Jarocki, J. Kostecki, J. Krzywinski, J. Mikołajczyk, R. Rakowski, A. Szczurek, and M. Szczurek, „Compact laser plasma EUV source based on a gas puff target for metrology applications", J. Alloys Compd. 401, 1-2, 99 (2005).

42. P.W. Wachulak, A. Bartnik, H. Fiedorowicz, P. Rudawski, R. Jarocki, J. Kostecki, and M. Szczurek, "Water window" compact, table-top laser plasma soft X-ray sources based on a gas puff target”, Nucl. Instr. Meth. B 268, 10, 1692-1700 (2010).

43. P.W. Wachulak, A. Bartnik, M. Skorupka, J. Kostecki, R. Jarocki, M. Szczurek, L. Wegrzynski, T. Fok and H. Fiedorowicz, "Water-window microscopy using a compact, laser-plasma SXR source based on a double-stream gas-puff target”, Appl. Phys. B 111, 2, 239-247 (2013).

44. P.W. Wachulak, A. Bartnik, L. Wegrzynski, J. Kostecki, R. Jarocki, T. Fok , M. Szczurek, and H. Fiedorowicz, "Sub $1-\mu \mathrm{m}$ resolution "water-window" microscopy using a compact, laser-plasma SXR source based on a double stream gas-puff target”, Nucl. Instr. Meth. B 311, 42-46 (2013).

45. R.L. Kelly, "Atomic and Ionic Spectrum Lines below 2000 Angstroms: Hydrogen through Krypton", J. Phys. Chem. Ref. Data 16, suppl. 1 (1987).

46. H. Wolter, "A Generalized Schwarschild Mirror Systems For Use at Glancing Incidence for X-ray Imaging", Ann. Physik 10, 286 (1952).

47. J.M. Heck, D.T. Attwood, W. Meyer-Ilse, and E.H. Anderson, "Resolution determination in X-ray microscopy: an analysis of the effects of partial coherence and illumination spectrum", J. X-Ray Sci. Technol. 8, 95-104 (1998).

48. D. Attwood, Soft X-Rays and Extreme Ultraviolet Radiation,Cambridge University, Cambridge, 1999.

49. P.W. Wachulak, A. Torrisi, A. Bartnik, D. Adjei, J. Kostecki, L. Wegrzynski, R. Jarocki, M. Szczurek, and H. Fiedorowicz, "Desktop water window microscope using a double-stream gas puff target source", Appl. Phys. B 118, 573-578 (2015).

50. A. Torrisi, P. Wachulak, F. Nawaz, A. Bartnik, J. Kostecki, L. Wegrzynski, R. Jarocki, M. Szczurek, and H. Fiedorowicz, „Nanoscale imaging and optimization of a compact "water window" SXR microscope", Proc. of SPIE 9510, 95100N95100N-11 (2015).

51. A. Torrisi, P. Wachulak, M. F. Nawaz, A. Bartnik, Ł. Węgrzyński, A. Jancarek, and H. Fiedorowicz, "Characterization and optimization of images acquired by a compact soft X-ray microscope based on a double stream gas-puff target source", J. Instrumentation, accepted for publication (2016).

52. CXRO X-Ray Interactions With Matter website: http://henke. lbl.gov/optical_constants/

53. P. Wachulak, A. Torrisi, M. F. Nawaz, A. Bartnik, D. Adjei, Š. Vondrová, J. Turňová, A. Jančarek, J. Limpouch, M. Vrbová, and H. Fiedorowicz, "A compact "Water Window" microscope with $60 \mathrm{~nm}$ spatial resolution for applications in biology and nanotechnology", Microscopy and Microanalysis 21, 1214-1223 (2015). 